\title{
Problems with Mixed Boundary Conditions in Banach Spaces
}

\author{
Dionicio Pastor Dallos Santos \\ Department of Mathematics, IME-USP, Cidade Universitária, 05508-090 São Paulo, SP, Brazil \\ Correspondence should be addressed to Dionicio Pastor Dallos Santos; dionicio@ime.usp.br
}

Received 22 November 2016; Accepted 22 February 2017; Published 15 March 2017

Academic Editor: Zhilin Yang

Copyright (C) 2017 Dionicio Pastor Dallos Santos. This is an open access article distributed under the Creative Commons Attribution License, which permits unrestricted use, distribution, and reproduction in any medium, provided the original work is properly cited.

Using Leray-Schauder degree or degree for $\alpha$-condensing maps we obtain the existence of at least one solution for the boundary value problem of the following type: $\left(\varphi\left(u^{\prime}\right)\right)^{\prime}=f\left(t, u, u^{\prime}\right), u(T)=0=u^{\prime}(0)$, where $\varphi: X \rightarrow X$ is a homeomorphism with reverse Lipschitz constant such that $\varphi(0)=0, f:[0, T] \times X \times X \rightarrow X$ is a continuous function, $T$ is a positive real number, and $X$ is a real Banach space.

\section{Introduction}

The purpose of this article is to obtain some existence results for the nonlinear boundary value problem of the form

$$
\begin{gathered}
\left(\varphi\left(u^{\prime}\right)\right)^{\prime}=f\left(t, u, u^{\prime}\right), \\
u(T)=0=u^{\prime}(0),
\end{gathered}
$$

where $\varphi: X \rightarrow X$ is a homeomorphism such that $\varphi(0)=0$ and $\varphi^{-1}$ is Lipschitz constant, $f:[0, T] \times X \times X \rightarrow X$ is a continuous function, $T$ is a positive real number, and $X$ is a real Banach space. Of course, a solution of this problem is a function $u:[0, T] \rightarrow X$ of class $C^{1}$ such that the function $t \mapsto \varphi\left(u^{\prime}(t)\right)$ is continuously differentiable, satisfying the boundary conditions, and $\left(\varphi\left(u^{\prime}(t)\right)\right)^{\prime}=f\left(t, u(t), u^{\prime}(t)\right)$ for all $t \in[0, T]$.

The existence of solutions for second-order boundary value problems has been studied by many authors using various methods (see [1-6]).

In particular, the authors in [3] have studied the following boundary value problem:

$$
\begin{aligned}
u^{\prime \prime} & =f\left(t, u, u^{\prime}\right), \\
a u(0)-b u^{\prime}(0) & =u_{0}, \\
c u(1)+d u^{\prime}(1) & =u_{1},
\end{aligned}
$$

where $a, b, c, d$, and $a d+b c>0$. They obtained the existence of solutions of (2) using Darbo fixed point theorem and properties of the measure of noncompactness.

Recently, Zhou and Peng [5] have studied the following boundary value problem:

$$
\begin{aligned}
& -u^{\prime \prime}=f(t, u), \\
& u(0)=0=u(1),
\end{aligned}
$$

where $f:[0,1] \times X \rightarrow X$ is a continuous function and $X$ is a Banach space. They obtained the existence of solutions of (3), where the main tools used in the study are Sadovskii fixed point theorem and precise computation of measure of noncompactness.

Inspired by these results, the main aim of this paper is to study the existence of at least one solution for the boundary value problem (1) using Leray-Schauder degree or degree for $\alpha$-condensing maps. For this, we reduce the nonlinear boundary value problem to some fixed points problem. Next, we shall essentially consider two types of regularity assumptions for $f(t, x, y)$. In Theorem 10 we suppose that $f$ is completely continuous, which allows us to prove that the associated fixed point operator is completely continuous required by a Leray-Schauder approach. In Theorem 11 we only assume some regularity conditions expressed in terms of the measure of noncompactness, which allows us to apply the methods of topological degree theory for $\alpha$-condensing maps. 
The paper is organized as follows. In Section 2, we establish the notation, terminology, and various lemmas which will be used throughout this paper. In Section 3, we formulate the fixed point operator equivalent to problem (1). In Section 4, we give main results in this paper. In Section 5, we study the existence of at least one solution for (1) in Hilbert spaces. For these results, we adapt the ideas of [7-9] to the present situation.

\section{Notations and Preliminary Results}

We first introduce some notation. For fixed $T$, we denote the usual norm in $L^{1}=L^{1}([0, T], X)$ by $\|\cdot\|_{L^{1}}$. Let $C=C([0, T], X)$ denote the Banach space of continuous functions from $[0, T]$ into $X$, endowed with the uniform norm $\|\cdot\|_{\infty}$ and let $C^{1}=C^{1}([0, T], X)$ denote the Banach space of continuously differentiable functions from $[0, T]$ into $X$, equipped with the usual norm $\|u\|_{1}=\max \left\{\|u\|_{\infty},\left\|u^{\prime}\right\|_{\infty}\right\}$.

We introduce the following applications:

The Nemytskii operator $N_{f}: C^{1} \rightarrow C$ is

$$
N_{f}(u)(t)=f\left(t, u(t), u^{\prime}(t)\right)
$$

the integration operator $H: C \rightarrow C^{1}$ is

$$
H(u)(t)=\int_{0}^{t} u(s) d s,
$$

and the continuous linear application $K: C \rightarrow C^{1}$ is

$$
K(u)(t)=-\int_{t}^{T} u(s) d s .
$$

Throughout this paper, we denote by $(X,\|\cdot\|)$ a real Banach space and $I=[0, T]$. For $A \subseteq C^{1}$, we use the notation

$$
\begin{aligned}
A(t) & =\{u(t): u \in A\}, \\
A(I) & =\{u(t): u \in A, t \in I\}, \\
A^{\prime}(t) & =\left\{u^{\prime}(t): u \in A\right\}, \\
A^{\prime} & =\left\{u^{\prime}: u \in A\right\}, \\
A^{\prime}(I) & =\left\{u^{\prime}(t): u \in A, t \in I\right\} .
\end{aligned}
$$

Definition 1. Let $X$ be a Banach space and let $M_{X}$ be the family of bounded subsets of $X$. The Kuratowski measure of noncompactness is the map $\alpha: M_{X} \rightarrow[0, \infty)$ defined for

$$
\begin{aligned}
& \alpha(B)=\inf [d \\
& \quad>0: B \text { admits a finite cover by sets of diameter } \\
& \quad \leq d] ; \text { here } B \in M_{X} .
\end{aligned}
$$

\section{Properties}

(a) $\alpha(B)=0$ iff $\bar{B}$ is compact. (b) $S \subset B$ then $\alpha(S) \leq \alpha(B)$.

(c) $\alpha(\bar{B})=\alpha(B)$.

(d) $\alpha(B \cup S)=\max \{\alpha(B), \alpha(S)\}$.

(e) $\alpha(\lambda B)=|\lambda| \alpha(B)$, where $\lambda \in \mathbb{R}$ and $\lambda B=\{\lambda b: b \in B\}$.

(f) $\alpha(B+S) \leq \alpha(B)+\alpha(S)$, where $B+S=\{b+s: b \in B, s \in$ $S\}$.

(g) $\alpha(\overline{\operatorname{conv}}(B))=\alpha(B)$.

The details of $\alpha$ and its properties can be found in [10].

Definition 2 (see [11]). Assuming that $D \subset X$ the mapping $A$ : $D \rightarrow X$ is said to be a condensing operator if $A$ is continuous and bounded (sends bounded sets into bounded sets), and, for any nonrelatively compact and bounded set $S \subset D$,

$$
\alpha(A(S))<\alpha(S) .
$$

The following lemmas are of great importance in the proof of our main results. The proofs can be found in [11].

In the following, we denote $\alpha_{c}$ and $\alpha_{1}$ as the noncompactness measure in $C$ and $C^{1}$, respectively.

Lemma 3. Let $S$ be a bounded subset of real numbers and $B$ a bounded subset of $X$. Then

$$
\alpha(S B)=\left(\sup _{t \in S}|t|\right) \alpha(B),
$$

where $S B=\{s b: s \in S, b \in B\}$.

Lemma 4. Let $A, B$ be bounded subsets of Banach spaces $X$ and $Y$, respectively, with

$$
\|(x, y)\|=\max \{\|x\|,\|y\|\} .
$$

Then

$$
\alpha(A \times B)=\max \{\alpha(A), \alpha(B)\} .
$$

Lemma 5. If $H \subset C$ is bounded and equicontinuous, then we have the following:

(1) $\alpha_{c}(H)=\alpha(H(I))$.

(2) $\alpha(H(I))=\max _{I} \alpha(H(t))$.

Lemma 6. If $H$ is a bounded set in $C^{1}$, then

(i) $\alpha_{1}(H) \geq \alpha(H(I))$,

(ii) $2 \alpha_{1}(H) \geq \alpha\left(H^{\prime}(I)\right)$.

Lemma 7. If $H$ is a bounded set in $C^{1}$ and $H^{\prime}$ equicontinuous, then

$$
\alpha_{1}(H)=\max \left\{\max _{I} \alpha(H(t)), \max _{I} \alpha\left(H^{\prime}(t)\right)\right\} .
$$

\section{Fixed Point Formulations}

Let us consider the operator

$$
\begin{aligned}
M_{1}: C^{1} & \longrightarrow C^{1}, \\
u & \longmapsto K\left(\varphi^{-1}\left[H\left(N_{f}(u)\right)\right]\right) .
\end{aligned}
$$


Here $\varphi^{-1}$ is understood as the operator $\varphi^{-1}: C \rightarrow C$ defined for $\varphi^{-1}(v)(t)=\varphi^{-1}(v(t))$. It is clear that $\varphi^{-1}$ is continuous and sends bounded sets into bounded sets.

Lemma 8. $u \in C^{1}$ is a solution of (1) if and only if $u$ is a fixed point of the operator $M_{1}$.

Proof. Let $u$ be a solution of (1). This implies that

$$
\begin{gathered}
\left(\varphi\left(u^{\prime}\right)\right)^{\prime}=f\left(t, u, u^{\prime}\right), \\
u(T)=0=u^{\prime}(0) .
\end{gathered}
$$

Integrating of 0 to $t$ and using the fact that $u^{\prime}(0)=0$, we deduce that

$$
\varphi\left(u^{\prime}(t)\right)=H\left(N_{f}(u)\right)(t)
$$

Applying $\varphi^{-1}$ and $K$ to both of its members and using that $u(T)=0$, we have that

$$
\begin{array}{r}
u(t)=K\left(\varphi^{-1}\left[H\left(N_{f}(u)\right)\right]\right)(t)=M_{1}(u)(t) \\
(t \in[0, T]) .
\end{array}
$$

Conversely, since, by definition of the mapping $M_{1}$,

$$
\begin{aligned}
K\left(\varphi^{-1}\left[H\left(N_{f}(u)\right)\right]\right)(T) & =0, \\
\varphi^{-1}\left[H\left(N_{f}(u)\right)\right](0) & =0,
\end{aligned}
$$

it is a simple matter to see that if $u$ is such that $u=M_{1}(u)$ then $u$ is a solution to (1).

Using the theorem of Arzelà-Ascoli we show that the operator $M_{1}$ is completely continuous.

Lemma 9. If $f$ is completely continuous, then the operator $M_{1}: C^{1} \rightarrow C^{1}$ is completely continuous.

Proof. Let $\Lambda \subset C^{1}$ be a bounded set. Then, if $u \in \Lambda$, there exists a constant $\rho>0$ such that

$$
\|u\|_{1} \leq \rho .
$$

Next, we show that $\overline{M_{1}(\Lambda)} \subset C^{1}$ is a compact set. Let $\left(v_{n}\right)_{n}$ be a sequence in $M_{1}(\Lambda)$, and let $\left(u_{n}\right)_{n}$ be a sequence in $\Lambda$ such that $v_{n}=M_{1}\left(u_{n}\right)$. Using (19), we have that there exists a constant $W>0$ such that, for all $n \in \mathbb{N}$,

$$
\left\|N_{f}\left(u_{n}\right)\right\|_{\infty} \leq W,
$$

which implies that

$$
\left\|H\left(N_{f}\left(u_{n}\right)\right)\right\|_{\infty} \leq W T \text {. }
$$

Hence the sequence $\left(H\left(N_{f}\left(u_{n}\right)\right)\right)_{n}$ is bounded in C. Moreover, for $t, t_{1} \in[0, T]$ and for all $n \in \mathbb{N}$, we have that

$$
\begin{aligned}
\| H & \left(N_{f}\left(u_{n}\right)\right)(t)-H\left(N_{f}\left(u_{n}\right)\right)\left(t_{1}\right) \| \\
= & \| \int_{0}^{t} f\left(s, u_{n}(s), u_{n}^{\prime}(s)\right) d s \\
& -\int_{0}^{t_{1}} f\left(s, u_{n}(s), u_{n}^{\prime}(s)\right) d s \| \\
& =\left\|\int_{t_{1}}^{t} f\left(s, u_{n}(s), u_{n}^{\prime}(s)\right) d s\right\| \leq W\left|t-t_{1}\right|,
\end{aligned}
$$

which implies that $\left(H\left(N_{f}\left(u_{n}\right)\right)\right)_{n}$ is equicontinuous.

On the other hand, for $t \in[0, T]$,

$$
B(t)=\left\{H\left(N_{f}\left(u_{n}\right)\right)(t): n \in \mathbb{N}\right\},
$$

where

$$
\begin{aligned}
H & \left(N_{f}\left(u_{n}\right)\right)(t)=\int_{0}^{t} N_{f}\left(u_{n}\right)(s) d s \\
& =\int_{0}^{t} f\left(s, u_{n}(s), u_{n}^{\prime}(s)\right) d s \\
& =t \lim _{m \rightarrow \infty} \sum_{k=1}^{m} f\left(s_{k}, u_{n}\left(s_{k}\right), u_{n}^{\prime}\left(s_{k}\right)\right) \frac{\left(s_{k}-s_{k-1}\right)}{t} .
\end{aligned}
$$

Recalling that the convex hull of a set $A \subseteq X$ is given by $\operatorname{conv}(A)$

$$
=\left\{\sum_{i=1}^{N} \alpha_{i} x_{i}: x_{i} \in A, \alpha_{i} \in \mathbb{R}, \alpha_{i} \geq 0, \sum_{i=1}^{N} \alpha_{i}=1\right\},
$$

it follows that

$$
\begin{aligned}
& \int_{0}^{t} f\left(s, u_{n}(s), u_{n}^{\prime}(s)\right) d s \\
& \quad \in t \overline{\operatorname{conv}}\left(\left\{f\left(s, u_{n}(s), u_{n}^{\prime}(s)\right): s \in[0, T], n \in \mathbb{N}\right\}\right),
\end{aligned}
$$

which implies that

$$
\begin{aligned}
& B(t)=\left\{H\left(N_{f}\left(u_{n}\right)\right)(t): n \in \mathbb{N}\right\} \\
& \quad C t \overline{\operatorname{conv}}\left(\left\{f\left(s, u_{n}(s), u_{n}^{\prime}(s)\right): s \in[0, T], n \in \mathbb{N}\right\}\right) .
\end{aligned}
$$

Using the fact that $f:[0, T] \times X \times X \rightarrow X$ is completely continuous, we deduce that $\alpha(B(t))=0$. Hence, $B(t)$ is a relatively compact set in $X$. Thus, by the Arzelà-Ascoli theorem there is a subsequence of $\left(H\left(N_{f}\left(u_{n}\right)\right)\right)_{n}$, which we call $\left(H\left(N_{f}\left(u_{n_{j}}\right)\right)\right)_{j}$, which is convergent in $C$. Using the fact that $\varphi^{-1}: C \rightarrow C$ is continuous it follows from

$$
M_{1}\left(u_{n_{j}}\right)^{\prime}=\varphi^{-1}\left[H\left(N_{f}\left(u_{n_{j}}\right)\right)\right]
$$


that the sequence $\left(M_{1}\left(u_{n_{j}}\right)^{\prime}\right)_{j}$ is convergent in $C$ and hence $\left(v_{n_{j}}\right)_{j}=\left(M_{1}\left(u_{n_{j}}\right)\right)_{j}$ is convergent in $C^{1}$. Finally, let $\left(v_{n}\right)_{n}$ be a sequence in $\overline{M_{1}(\Lambda)}$. Let $\left(z_{n}\right)_{n} \subseteq M_{1}(\Lambda)$ be such that

$$
\lim _{n \rightarrow \infty}\left\|z_{n}-v_{n}\right\|_{1}=0 .
$$

Let $\left(z_{n_{j}}\right)_{j}$ be a subsequence of $\left(z_{n}\right)_{n}$ such that it converges to $z$. It follows that $z \in \overline{M_{1}(\Lambda)}$ and $\left(v_{n_{j}}\right)_{j}$ converge to $z$. This concludes the proof.

In order to apply Leray-Schauder degree to the operator $M_{1}$, we introduced a family of problems depending on a parameter $\lambda$. For $\lambda \in[0,1]$, we consider the family of boundary value problems

$$
\begin{gathered}
\left(\varphi\left(u^{\prime}\right)\right)^{\prime}=\lambda f\left(t, u(t), u^{\prime}(t)\right) \\
u(T)=0=u^{\prime}(0) .
\end{gathered}
$$

Notice that (30) coincide, for $\lambda=1$, with (1). So, for each $\lambda \in[0,1]$, the operator associated with (30) for Lemma 8 is the operator $M(\lambda, \cdot)$, where $M$ is defined on $[0,1] \times C^{1}$ by

$$
M(\lambda, u)=K\left(\varphi^{-1}\left[\lambda H\left(N_{f}(u)\right)\right]\right) .
$$

Using the same arguments as in the proof of Lemma 9 we show that the operator $M$ is completely continuous. Moreover, using the same reasoning as above, system (30) (see Lemma 8) is equivalent to the problem

$$
u=M(\lambda, u) .
$$

\section{Main Results}

In this section, we present and prove our main results.

Theorem 10. Let $X$ be a Banach space and $\varphi^{-1}$ a homeomorphism with Lipschitz constant $k$. Suppose that $f$ is completely continuous and that there exist two numbers $c_{0}, c_{1} \geq 0$ such that

$$
\begin{aligned}
&\|f(t, x, y)\| \leq c_{0}+c_{1}\|y\|, \\
& \forall(t, x, y) \in[0, T] \times X \times X .
\end{aligned}
$$

Then problem (1) has at least one solution.

Proof. Let $(\lambda, u) \in[0,1] \times C^{1}$ be such that $M(\lambda, u)=u$. Using (32) we have that $u$ is solution of (30), which implies that

$$
\begin{aligned}
u^{\prime} & =\varphi^{-1}\left[\lambda H\left(N_{f}(u)\right)\right], \\
u^{\prime}(0) & =0=u(T) .
\end{aligned}
$$

Using the fact that $\varphi^{-1}$ is a homeomorphism with Lipschitz constant $k$, we deduce that

$$
\left\|u^{\prime}(t)\right\| \leq k c_{0} T+k c_{1} \int_{0}^{t}\left\|u^{\prime}(s)\right\| d s \quad(t \in[0, T]) .
$$

By Gronwall's Inequality, we have

$$
\left\|u^{\prime}(t)\right\| \leq k c_{0} T e^{\int_{0}^{t} k c_{1} d s} \leq k c_{0} T e^{k c_{1} T} \quad(t \in[0, T]) .
$$

Hence, $\left\|u^{\prime}\right\|_{\infty} \leq k c_{0} T e^{k c_{1} T} \quad \beta$. Because $u \in C^{1}$ is such that $u^{\prime}(T)=0$, we have that

$$
\|u(t)\| \leq \int_{t}^{T}\left\|u^{\prime}(s)\right\| d s \leq \int_{0}^{T}\left\|u^{\prime}(s)\right\| d s \leq \beta T
$$

$$
(t \in[0, T]),
$$

and hence

$$
\|u\|_{1} \leq R_{1}, \quad \text { where } R_{1}=\max \{\beta, \beta T\} .
$$

Using that $M$ is completely continuous we deduce that, for each $\lambda \in[0,1]$, the Leray-Schauder degree $\operatorname{deg}_{\text {LS }}(I-$ $\left.M(\lambda, \cdot), B_{\rho}(0), 0\right)$ is well-defined for any $\rho>R_{1}$, and by the homotopy invariance we have that

$$
\begin{aligned}
& \operatorname{deg}_{\mathrm{LS}}\left(I-M(1, \cdot), B_{\rho}(0), 0\right) \\
& \quad=\operatorname{deg}_{\mathrm{LS}}\left(I-M(0, \cdot), B_{\rho}(0), 0\right) .
\end{aligned}
$$

Hence, $\operatorname{deg}_{\mathrm{LS}}\left(I-M(1, \cdot), B_{\rho}(0), 0\right) \neq 0$. This, in turn, implies that there exists $u \in B_{\rho}(0)$ such that $M_{1}(u)=u$, which is a solution for (1).

Theorem 11. Let $X$ be a Banach space and $\varphi^{-1}$ a homeomorphism with Lipschitz constant $k$. Assume that $f$ is continuous and satisfies the following conditions:

(1) There exist two numbers $c_{0}, c_{1} \geq 0$ such that

$$
\begin{aligned}
\|f(t, x, y)\| \leq c_{0}+c_{1}\|y\|, & \\
& \forall(t, x, y) \in[0,1] \times X \times X .
\end{aligned}
$$

(2) For all bounded subsets $A, B$ in $X$,

$$
\begin{aligned}
& \alpha(f([0,1] \times A \times B)) \leq k_{1} \max \{\alpha(A), \alpha(B)\}, \\
& \text { where } 0<k_{1}<\frac{1}{2 k} .
\end{aligned}
$$

Then problem (1) has at least one solution.

Proof. Observe that $M_{1}$ maps bounded sets into bounded sets. Furthermore, its continuity follows by the continuity of the operators which compose $M_{1}$. We show that the operator $M_{1}$ is condensing ( $\alpha$-condensing). In fact, for a bounded set $\Lambda$ in $C^{1}$, there exists a constant $L_{1}>0$ such that

$$
\left\|N_{f}(u)\right\|_{\infty} \leq L_{1}, \quad \forall u \in \Lambda .
$$

For $t, t_{1} \in[0,1]$ we have that

$$
\begin{aligned}
& \left\|\left(M_{1} u\right)^{\prime}(t)-\left(M_{1} u\right)^{\prime}\left(t_{1}\right)\right\| \\
& \quad=\left\|\varphi^{-1}\left[H\left(N_{f}(u)\right)\right](t)-\varphi^{-1}\left[H\left(N_{f}\left(u_{n}\right)\right)\right]\left(t_{1}\right)\right\| \\
& \leq k\left\|H\left(N_{f}(u)\right)(t)-H\left(N_{f}(u)\right)\left(t_{1}\right)\right\| \\
& \leq k\left\|\int_{t_{1}}^{t} f\left(s, u(s), u^{\prime}(s)\right) d s\right\| \leq k L_{1}\left|t-t_{1}\right|,
\end{aligned}
$$


which means $\left(M_{1} \Lambda\right)^{\prime}$ is equicontinuous. Applying Lemma 7 there exists $\tau \in[0,1]$ or $\omega \in[0,1]$ with

$$
\alpha_{1}\left(M_{1} \Lambda\right)=\alpha\left(\left(M_{1} \Lambda\right)(\tau)\right)
$$

or

$$
\alpha_{1}\left(M_{1} \Lambda\right)=\alpha\left(\left(M_{1} \Lambda\right)^{\prime}(\omega)\right) .
$$

Let us consider the first case.

$$
\begin{aligned}
& \alpha_{1}\left(M_{1} \Lambda\right)=\alpha\left(\left(M_{1} \Lambda\right)(\tau)\right) \\
& \quad=\alpha\left(\left\{K\left(\varphi^{-1}\left[H\left(N_{f}(u)\right)\right]\right)(\tau): u \in \Lambda\right\}\right) .
\end{aligned}
$$

Using the properties of $\alpha$, we see that

$$
\begin{aligned}
& \alpha_{1}\left(M_{1} \Lambda\right) \leq \alpha\left(\left\{\int_{\tau}^{1} \varphi^{-1}\left[H\left(N_{f}(u)\right)(s)\right] d s: u\right.\right. \\
& \in \Lambda\}) \leq(1-\tau) \alpha\left(\overline { \operatorname { c o n v } } \left\{\varphi^{-1}\left[H\left(N_{f}(u)\right)(s)\right]: s\right.\right. \\
& \quad \in[\tau, 1], u \in \Lambda\}) \leq \alpha\left(\left\{\varphi^{-1}\left[H\left(N_{f}(u)\right)(s)\right]: s\right.\right. \\
& \quad \in[0,1], u \in \Lambda\}) .
\end{aligned}
$$

Using the fact that $\varphi^{-1}$ is a homeomorphism with Lipschitz constant $k$, we deduce that

$$
\begin{aligned}
& \alpha_{1}\left(M_{1} \Lambda\right) \leq k \alpha\left(\left\{H\left(N_{f}(u)\right)(s): s \in[0,1], u \in \Lambda\right\}\right) \\
& \quad \leq k \alpha\left(\left\{\int_{0}^{s} f\left(t, u(t), u^{\prime}(t)\right) d t: s \in[0,1], u\right.\right. \\
& \in \Lambda\}) \leq k \alpha\left([ 0 , 1 ] \overline { \operatorname { c o n v } } \left\{f\left(t, u(t), u^{\prime}(t)\right): t\right.\right. \\
& \quad \in[0,1], u \in \Lambda\}) .
\end{aligned}
$$

Applying Lemma 3 and again the properties of $\alpha$, we obtain that

$$
\begin{aligned}
& \alpha_{1}\left(M_{1} \Lambda\right) \\
& \quad \leq k \alpha\left(\left\{f\left(t, u(t), u^{\prime}(t)\right): t \in[0,1], u \in \Lambda\right\}\right) \\
& \quad \leq k \alpha\left(f\left([0,1] \times \Lambda([0,1]) \times \Lambda^{\prime}([0,1])\right)\right) .
\end{aligned}
$$

Using (41), we have that

$$
\alpha_{1}\left(M_{1} \Lambda\right) \leq k k_{1} \max \left\{\alpha(\Lambda([0,1])), \alpha\left(\Lambda^{\prime}([0,1])\right)\right\} .
$$

This implies, by Lemma 6, that

$$
\alpha_{1}\left(M_{1} \Lambda\right) \leq 2 k k_{1} \alpha_{1}(\Lambda) .
$$
obtain

Consider the alternative case. Proceeding as before, we

$$
\alpha_{1}\left(M_{1} \Lambda\right)=\alpha\left(\left(M_{1} \Lambda\right)^{\prime}(\omega)\right) \leq 2 k k_{1} \alpha_{1}(\Lambda) .
$$

Therefore, in either case, we obtain

$$
\alpha_{1}\left(M_{1} \Lambda\right) \leq 2 k k_{1} \alpha_{1}(\Lambda) .
$$

By (41), we get $0<2 k k_{1}<1$, and therefore $M_{1}$ is $\alpha$ condensing.

Let us consider the function

$$
\begin{aligned}
\widetilde{M}:[0,1] \times C^{1} & \longrightarrow C^{1}, \\
(\lambda, u) & \longmapsto \lambda M_{1}(u) .
\end{aligned}
$$

Let $(\lambda, u) \in[0,1] \times C^{1}$ be such that $u=\widetilde{M}(\lambda, u)$. Using the fact that $\varphi^{-1}$ is a homeomorphism with Lipschitz constant $k$ and Gronwall's Inequality, we deduce that there exists a constant $r>0$ such that $\|u\|_{1}<r$.

Finally, we show the existence of at least one solution of (1) using the homotopy invariance of the degree for $\alpha$ condensing maps. Let $B$ be bounded in $C^{1}$. Then

$$
\begin{aligned}
\alpha_{1} & (\widetilde{M}([0,1] \times B)) \\
\quad & \alpha_{1}(\widetilde{M}(\lambda, u): \lambda \in[0,1], u \in B) \leq 2 k k_{1} \alpha_{1}(B) .
\end{aligned}
$$

Then we have that, for each $\lambda \in[0,1]$, the degree $\operatorname{deg}_{N}(I-$ $\left.\widetilde{M}(\lambda, \cdot), B_{r}(0), 0\right)$ is well-defined and, by the properties of that degree, that

$$
\begin{aligned}
& \operatorname{deg}_{N}\left(I-\widetilde{M}(1, \cdot), B_{r}(0), 0\right) \\
& \quad=\operatorname{deg}_{N}\left(I-\widetilde{M}(0, \cdot), B_{r}(0), 0\right)=1 .
\end{aligned}
$$

Then, from the existence property of degree, there exists $u \in$ $B_{r}(0)$ such that $u=\widetilde{M}(1, u)=M_{1}(u)=u$, which is a solution for (1).

Remark 12. In [3], the nonlinear term $f(t, x, y)$ is bounded; in our result, the nonlinear term $f(t, x, y)$ may possess no more than linear growth.

Now we consider an example to illustrate our results.

Example 13. Consider the boundary value problem in

$$
X=l^{\infty}=\left\{x=\left(x_{1}, x_{2}, \ldots, x_{i}, \ldots\right): \sup _{i}\left|x_{i}\right|<\infty\right\},
$$

with norm $\|x\|=\sup _{i}\left|x_{i}\right|$.

$$
\begin{aligned}
u_{i}^{\prime \prime} & =\frac{\cos \left(u_{i}\right)}{i^{2}}+\frac{u_{i}^{\prime}}{4 e^{t}} \quad t \in[0,1], \\
u_{i}(1) & =u_{i}^{\prime}(0)=0 .
\end{aligned}
$$

Now we can regard this problem as a problem of form (1), where $u=\left(u_{1}, u_{2}, \ldots, u_{i}, \ldots\right) \in l^{\infty}$ and $f=$ $\left(f_{1}, f_{2}, \ldots, f_{i}, \ldots\right)$ with

$$
\begin{aligned}
f_{i}(t, x, y) & =g_{i}(t, x, y)+h_{i}(t, x, y) \\
& =\frac{\cos \left(x_{i}\right)}{i^{2}}+\frac{y_{i}}{4 e^{t}} \\
& (t, x, y) \in[0,1] \times l^{\infty} \times l^{\infty} .
\end{aligned}
$$


It is clear that $f:[0,1] \times l^{\infty} \times l^{\infty} \rightarrow l^{\infty}$ is a continuous function and that

$$
\|f(t, x, y)\|=\sup _{i}\left|f_{i}(t, x, y)\right| \leq 1+\|y\| .
$$

On the other hand, for any bounded subsets $A, B \subset l^{\infty}$ we have

$$
\begin{aligned}
& \alpha(g([0,1] \times A \times B))=0, \\
& \alpha(h([0,1] \times A \times B))=\frac{\alpha(B)}{4},
\end{aligned}
$$

and hence

$$
\begin{gathered}
\alpha(f([0,1] \times A \times B)) \leq \frac{1}{4} \alpha(B) \\
\leq \frac{1}{4} \max \{\alpha(A), \alpha(B)\} .
\end{gathered}
$$

So, by Theorem 11 we get one solution.

\section{Boundary Value Problems in Hilbert Spaces}

Throughout this section, let $(X,\langle\cdot, \cdot\rangle)$ denote a real Hilbert space. Assume that $\varphi: X \rightarrow X$ satisfies the following conditions:

(1) $\varphi^{-1}$ is a homeomorphism with Lipschitz constant $k$.

(2) For any $x, y \in X, x \neq y$,

$$
\langle\varphi(x)-\varphi(y), x-y\rangle>0 .
$$

Lemma 14. Let $h \in C\left([0, T], \mathbb{R}^{+}\right)$be such that

$$
\|f(t, x, y)\| \leq\langle f(t, x, y), x\rangle+h(t)
$$

for all $(t, x, y) \in[0, T] \times X \times X$. If $(\lambda, u) \in[0,1] \times C^{1}$ is such that $M(\lambda, u)=u$, then there exists $R>0$ such that $\|u\|_{1} \leq R$.

Proof. Let $(\lambda, u) \in(0,1] \times C^{1}$ be such that $M(\lambda, u)=u$. Using (32), we have that $u$ is solution of (30), which implies that

$$
\begin{aligned}
u^{\prime} & =\varphi^{-1}\left[\lambda H\left(N_{f}(u)\right)\right], \\
u^{\prime}(0) & =0=u(T),
\end{aligned}
$$

where, for all $t \in[0, T]$, we obtain

$$
\begin{gathered}
\left\|\lambda H\left(N_{f}(u)\right)(t)\right\| \leq \int_{0}^{T}\left\|f\left(s, u(s), u^{\prime}(s)\right)\right\| d s \\
\quad \leq \int_{0}^{T}\left\langle f\left(s, u(s), u^{\prime}(s)\right), u(s)\right\rangle d s+\int_{0}^{T} h(s) d s .
\end{gathered}
$$

On the other hand, because $\varphi$ is a homeomorphism such that

$$
\langle\varphi(y), y\rangle \geq 0
$$

for all $y \in X$, then

$$
\left\langle\varphi\left(u^{\prime}(t)\right), u^{\prime}(t)\right\rangle \geq 0 \quad(t \in[0, T]),
$$

and hence

$$
-\int_{0}^{T}\left\langle\varphi\left(u^{\prime}(t)\right), u^{\prime}(t)\right\rangle d t \leq 0 .
$$

Using the integration-by-parts formula and the boundary conditions, we deduce that

$$
\begin{aligned}
& \int_{0}^{T}\left\langle\left(\varphi\left(u^{\prime}(t)\right)\right)^{\prime}, u(t)\right\rangle d t \\
& \quad=-\int_{0}^{T}\left\langle\varphi\left(u^{\prime}(t)\right), u^{\prime}(t)\right\rangle d t \leq 0 .
\end{aligned}
$$

Since $\lambda \in(0,1]$ and $u$ is solution of $(30)$ we have that

$$
\int_{0}^{T}\left\langle f\left(t, u(t), u^{\prime}(t)\right), u(t)\right\rangle d t \leq 0 .
$$

Hence,

$$
\left\|\varphi\left(u^{\prime}(t)\right)\right\| \leq\|h\|_{L^{1}}
$$

It follows that there exists $L>0$ such that $\left\|u^{\prime}\right\|_{\infty} \leq L$. Because $u \in C^{1}$ is such that $u(T)=0$, we deduce that

$$
\|u(t)\| \leq \int_{t}^{T}\left\|u^{\prime}(s)\right\| d s \leq \int_{0}^{T}\left\|u^{\prime}(s)\right\| d s \leq L T
$$

$$
(t \in[0, T]),
$$

and hence

$$
\|u\|_{1} \leq R, \quad \text { where } R=\max \{L, L T\} .
$$

Finally, if $u=M(0, u)$, then $u=0$, so the proof is complete.

Now we show the existence of at least one solution for problem (1) by means of Leray-Schauder degree.

Theorem 15. Let $f$ be completely continuous. Assume that $f$ satisfies the conditions of Lemma 14. Then (1) has at least one solution.

Proof. Let $\lambda \in[0,1]$ and $u$ be a possible fixed point of $M(\lambda, \cdot)$. Then, using Lemma 14 we deduce that

$$
\|u\|_{1} \leq R, \quad \text { where } R=\max \{L, L T\} .
$$

Therefore, if $\rho>R$, it follows from the homotopy invariance of Leray-Schauder degree that $\operatorname{deg}_{\mathrm{LS}}\left(I-M(\lambda, \cdot), B_{\rho}(0), 0\right)$ is independent of $\lambda \in[0,1]$ so that if we notice that $M(0, \cdot)=0$,

$$
\begin{aligned}
& \operatorname{deg}_{\mathrm{LS}}\left(I-M(1, \cdot), B_{\rho}(0), 0\right) \\
& \quad=\operatorname{deg}_{\mathrm{LS}}\left(I-M(0, \cdot), B_{\rho}(0), 0\right)=1 .
\end{aligned}
$$

Hence there exists $u \in B_{\rho}(0)$ such that it is a solution for (1).

Using a proof similar to that of Theorem 11, we obtain the following existence result. 
Theorem 16. Let $f=f(t, x)$ be continuous. Assume that $f$ satisfies the following conditions:

(1) There exists $h \in C\left([0,1], \mathbb{R}^{+}\right)$such that

$$
\|f(t, x)\| \leq\langle f(t, x), x\rangle+h(t)
$$

for all $(t, x) \in[0,1] \times X$.

(2) $f$ sends bounded sets into bounded sets.

(3) For any bounded set $S$ in $X$,

$$
\alpha(f([0,1] \times S)) \leq k_{1} \alpha(S), \quad \text { where } 0<k_{1}<\frac{1}{k} .
$$

Then problem (1) has at least one solution.

Proof. Let $A$ be bounded in $C^{1}$. Applying Lemma 7 there exists $\tau \in[0,1]$ or $\omega \in[0,1]$ with

$$
\alpha_{1}(M([0,1] \times A))=\alpha(M([0,1] \times A)(\tau))
$$

or

$$
\alpha_{1}(M([0,1] \times A))=\alpha\left((M([0,1] \times A))^{\prime}(\omega)\right) .
$$

Proceeding as Theorem 11, we obtain in either case

$$
\alpha_{1}(M([0,1] \times A)) \leq k k_{1} \alpha_{1}(A), \quad \text { where } k k_{1}<1 .
$$

Using the homotopy invariance of the degree for $\alpha$ condensing maps, we obtain

$$
\begin{aligned}
& \operatorname{deg}_{N}\left(I-M(0, \cdot), B_{\rho}(0), 0\right)=\operatorname{deg}_{N}\left(I, B_{\rho}(0), 0\right) \\
& \quad=1, \quad \text { where } \rho>L .
\end{aligned}
$$

Then, from the existence property of degree, there exists $u \in$ $B_{\rho}(0)$ such that $u=M(1, u)$, which is a solution for (1).

The following corollary is concerned with the existence of one solution for (1).

Corollary 17. Assume that $f=f(t, x)$ satisfies the following conditions:

(1) Suppose that for any $\delta>0$ the mapping $f:[0,1] \times$ $X \rightarrow X$ is bounded and uniformly continuous in $[0,1] \times \overline{B_{\delta}(0)}$, where $\overline{B_{\delta}(0)}=\{x \in X:\|x\| \leq \delta\}$.

(2) There exists $h \in C\left([0,1], \mathbb{R}^{+}\right)$such that

$$
\|f(t, x)\| \leq\langle f(t, x), x\rangle+h(t)
$$

for all $(t, x) \in[0,1] \times X$.

(3) There exists a constant $k_{1}$ with $0<k_{1}<1 / k$ such that

$$
\begin{aligned}
\|f(t, x)-f(t, y)\| \leq k_{1}\|x-y\| & \\
& \forall(t, x) \in[0,1] \times X .
\end{aligned}
$$

Then problem (1) has at least one solution.
Proof. Let $S$ be a bounded set in $X$. Let us consider

$$
H=\left\{\psi_{x}: x \in S\right\}
$$

$$
\text { where } \psi_{x}(t)=f(t, x) \forall t \in[0,1] .
$$

Cleary, $H \subset C, H$ is bounded and equicontinuous. Thus, by using the conclusion of Lemma 5, we have

$$
\begin{aligned}
\alpha_{c}(H) & =\alpha(H([0,1]))=\alpha(f([0,1] \times S)) \\
& =\max _{[0,1]} \alpha(\{f(t, x): x \in S\}) .
\end{aligned}
$$

Using (84), we obtain

$$
\alpha(f([0,1] \times S)) \leq k_{1} \alpha(S) .
$$

By using the arguments of Theorem 16, we can obtain the conclusion of Corollary 17.

\section{Conflicts of Interest}

The author declares that there are no conflicts of interest regarding the publication of this article.

\section{Acknowledgments}

This research was supported by CAPES and CNPq/Brazil.

\section{References}

[1] J. Andres, L. Malaguti, and M. Pavlačková, "On second-order boundary value problems in Banach spaces: a bound sets approach," Topological Methods in Nonlinear Analysis, vol. 37, no. 2, pp. 303-341, 2011.

[2] J. Andres, L. Malaguti, and M. Pavlačková, "A Scorza-Dragoni approach to second-order boundary value problems in abstract spaces," Applied Mathematics \& Information Sciences, vol. 6, no. 2, pp. 177-192, 2012.

[3] J. Chandra, V. Lakshmikantham, and A. R. Mitchell, "Existence of solutions of boundary value problems for nonlinear secondorder systems in a Banach space," Nonlinear Analysis: Theory, Methods \& Applications, vol. 2, no. 2, pp. 157-168, 1978.

[4] D. P. D. Santos, "Existence of solutions to nonlinear problems with three-point boundary conditions," Electronic Journal of Differential Equations, vol. 35, pp. 1-10, 2017.

[5] W.-X. Zhou and J. Peng, "Existence of solution to a secondorder boundary value problem via noncompactness measures," Discrete Dynamics in Nature and Society, vol. 2012, Article ID 786404, 16 pages, 2012.

[6] P. Zhang, "Existence of positive solutions for nonlocal secondorder boundary value problem with variable parameter in Banach spaces," Fixed Point Theory and Applications, vol. 2011, article no. 43, 2011.

[7] C. Bereanu and J. Mawhin, "Boundary-value problems with non-surjective $\phi$-Laplacian and one-sided bounded nonlinearity," Advances in Differential Equations, vol. 11, no. 1, pp. 35-60, 2006.

[8] V. Bouchez and J. Mawhin, "Boundary value problems for a class of first order quasilinear ordinary differential equations," Portugaliae Mathematica, vol. 71, no. 3-4, pp. 217-247, 2014. 
[9] R. Manásevich and J. Mawhin, "Periodic solutions for nonlinear systems with $p$-Laplacian-like operators," Journal of Differential Equations, vol. 145, no. 2, pp. 367-393, 1998.

[10] K. Deimling, Nonlinear Functional Analysis, Springer, Berlin, Germany, 1985.

[11] D. Guo, Y. J. Cho, and J. Zhu, Partial Ordering Methods in Nonlinear Problems, Hauppauge, New York, NY, USA, 2004. 


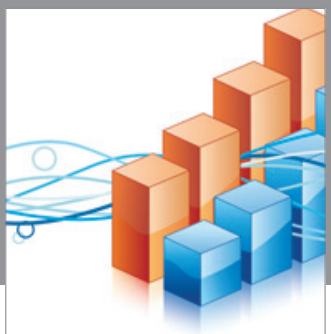

Advances in

Operations Research

vatem alat4

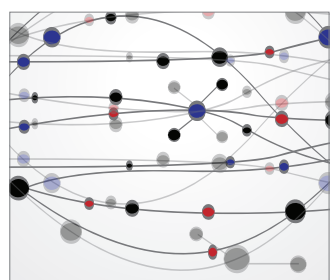

\section{The Scientific} World Journal
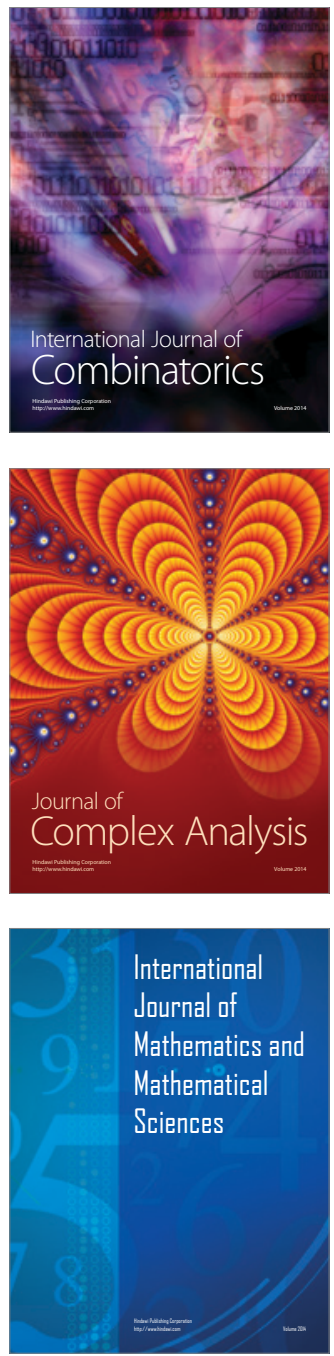
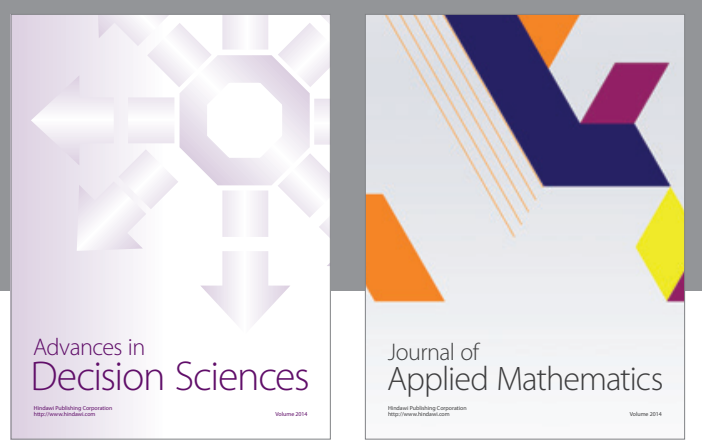

Algebra

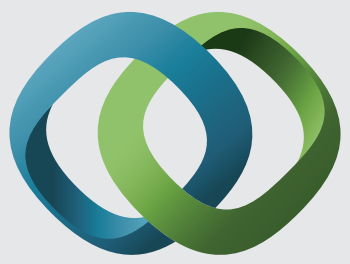

\section{Hindawi}

Submit your manuscripts at

https://www.hindawi.com
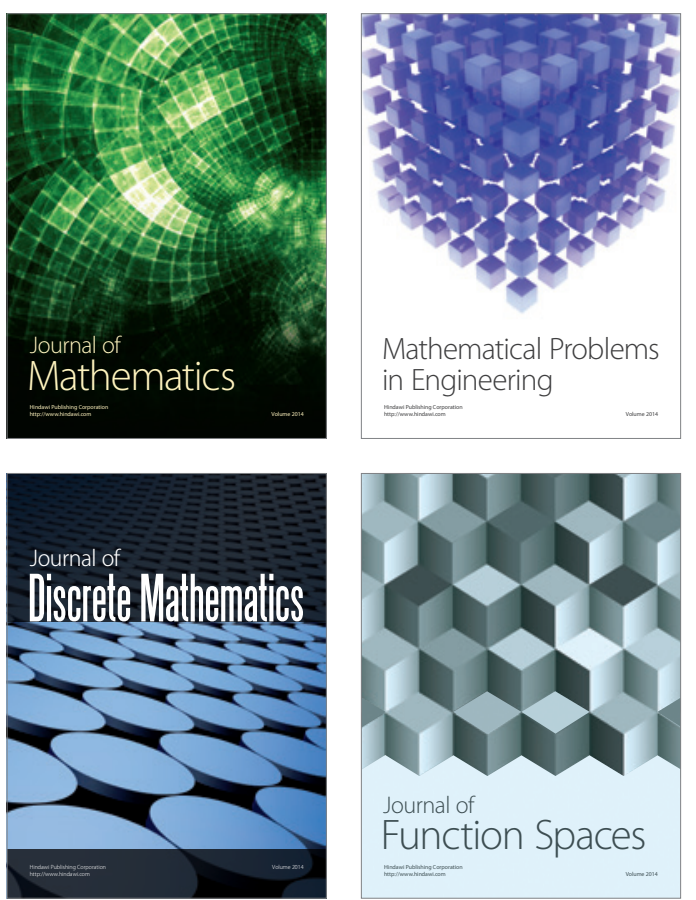

Mathematical Problems in Engineering
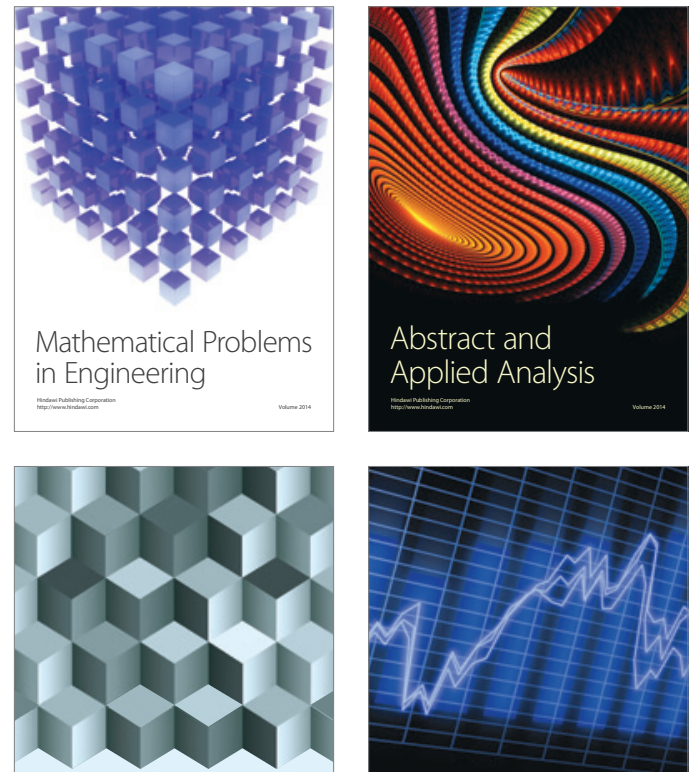

Journal of

Function Spaces

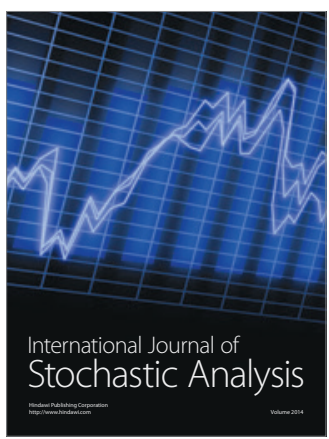

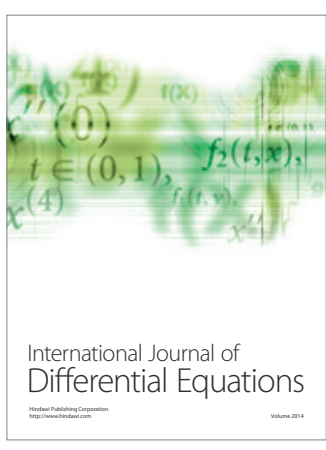
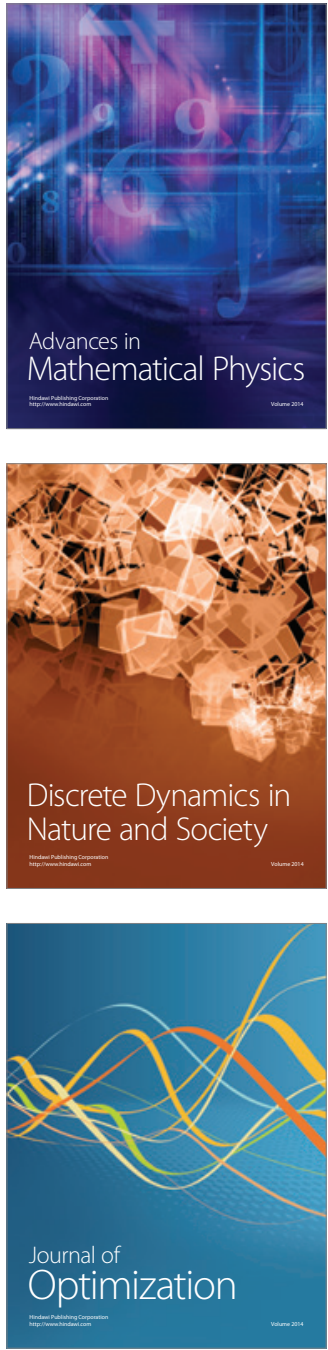